\title{
Computation of the Wind by Forced Adjustment to the Height Field ${ }^{1}$
}

\author{
FEdOR MESINGeR ${ }^{2}$ \\ Dept. of Meteorology, Universily of California, Los Angeles
}

(Manuscript received 23 July 1971, in revised form 28 September 1971)

\begin{abstract}
The problem of the forced adjustment of the wind field to the height field is experimentally studied with the Mintz:-Arakawa two-level atmospheric general circulation model.

In all but one of the experiments, the height field was assumed to be perfectly observed at 6-hr intervals, over a time period of one day or less, and from this height data the vector wind field was computed by forced dynamical adjustment. In one experiment, the temperature alone was prescribed. The winds computed in these experiments were compared with the "control" winds of the general circulation simulation.

The best agreement between the computed and the control winds was obtained when the time-differencing scheme in the governing finite-difference equations of motion had a large rate of damping of highfrequency motions. This damping rate also determined the optimum fraction and frequency of restoration of the height (or temperature) fields. With strong damping, total restoration every time step gave the most rapid rate of wind error reduction and the smallest asymptotic limit of the wind error.

The information content of the height field and its time derivatives was analyzed. The first time derivative of the height field was of much greater importance than the next higher time derivatives. In middle latitudes, where the time variation of the height field was large, the first time derivative reduced the computed wind error to about half of the error when using no time derivative. When the information is limited to $24 \mathrm{hr}$ or less, the total height field information (surface pressure as well as temperature) produced a much smaller wind error than temperature information alone.

With the first time derivative of the height field, the asymptotic limit of the computed wind error was about $1-1.5 \mathrm{~m} \mathrm{sec}^{-1}$ in middle latitudes and about $2.5 \mathrm{~m} \mathrm{sec}^{-1}$ in the tropics.
\end{abstract}

\section{Classification of past work and existing problems}

Numerical weather prediction is the solution of an initial-value, boundary-value problem in which the future state of the atmosphere depends on the initial distribution of the atmospheric state variables. When using the primitive equations of atmospheric motion, these variables are wind, temperature, "reference" pressure, and atmospheric composition (especially water vapor).

In the First GARP Global Experiment (FGGE), scheduled for the middle of this decade, an attempt will be made to measure the atmospheric state variables in the global domain and thereby obtain a "data set" for the experimental testing of various numerical weather prediction models (WMO-ICSU: GARP Publication Series and Special Reports, 1969-70).

But it is already clear (WMO-ICSU, 1969) that the FGGE will be deficient in observing the global wind field. In many regions direct observations of the wind will be missing or will not have the required accuracy. But in these regions we may still be able to determine

${ }^{I}$ UCLA Department of Meteorology Contribution No. 214. This research was supported by the National Aeronautics and Space Administration, under Grant NGR-05-007-091.

2 Present affiliation: Department of Meteorology, University of Belgrade, Yugoslavia. the height field (given observations of the temperature and a reference pressure), and from this height field we may be able to calculate an initial wind to the accuracy required. Conceivably, it may also be possible to calculate the initial wind from temperature observations only. Therefore, we should determine how accurately the wind can be calculated from the height field, or just the temperature field alone, and find the best procedure for doing this.

It is likely that in some regions the reverse requirement will exist, that is, a need to calculate the height field from the observed wind field. The same method may be applicable to this reverse problem.

A number of studies have been made in which either the wind was diagnostically calculated from some form of the balance equation and then used for numerical prediction with the primitive equations of motion; or in which the primitive equations themselves were used to calculate the initial wind by a forced dynamical adjustment to the height field or to the temperature field alone. These, as well as other initialization studies, can be classified according to whether 1 ) both the observed data and the calculation method are three-dimensional (for example, when solving the balance equation); 2) the observed data is three-dimensional and the calculation method is four-dimensional (Nitta and 
Hovermale, 1967; Miyakoda and Moyer, 1968); and 3) both the observed data and the calculation method are four-dimensional (Nitta and Hovermale, 1967; Charney et al., 1969).

The calculation of missing data is related to other problems in the initialization of the atmospheric state for numerical weather prediction.

The first of these problems is the spurious noise that is generated even with relatively small errors in the observed height or wind fields. Numerical prediction should improve if this noise is eliminated by an initial mutual dynamical adjustment of the fields.

Second, even if there are no observational errors, the space-time averaging performed by the particular observation method used may not be consistent with that implied by the equations of the numerical model.

Third, even if the averaging is properly done, the wind and the height fields need to be adjusted to obtain truncation error consistency in the particular model that will be used for the prediction.

Fourth, the information required at the grid points of each particular numerical model must be transferred there from the points at which the heights and the winds are observed.

Finally, the wind and the height fields must be brought into mutual adjustment at the single time, from observations that are nonsynchronous.

\section{Purpose of the present study}

Recent experience indicates that the most promising method of calculating the missing data is through fourdimensional data assimilation. This assimilation in its simplest form consists of carrying on the numerical integration while substituting the observed variables at appropriate times and places. During such integration, unobserved or poorly observed variables are adjusted by the geostrophic adjustment process, by artificial damping in the time differencing, and possibly by other processes. An advantage of this procedure is the consistency of the truncation errors of the initialization and the forecast (Nitta and Hovermale, 1969), and the simplicity of the programing required.

The reported experimental results, as far as the basic physical possibilities of such an approach are concerned, are very encouraging. Charney et al. (1969) obtained a wind field with a very small rms difference from that of a long simulation run. Even with moderate random errors in the temperature field, and no surface pressure data, they achieved a wind field with an rms error of less than $1 \mathrm{~m} \mathrm{sec}^{-1}$.

A serious remaining problem in this type of initialization is the uncertainty about the performance of the method when using real data, data which is alien to the numerical model. It appears that a given method can accomplish a particular time rate of adjustment of the fields, and that this rate, in addition, is about proportional to the amount of the imbalance present. On the other hand, there is also a certain time rate of growth of the mathematical and physical errors in the numerical integration. When, in an initialization run, the adjustment of the fields becomes sufficiently good for the adjustment rate to decrease to about the level of the rate of growth of the mathematical and physical errors, not much further adjustment can take place. Thus, when using simulated observations generated by the same numerical model, which produce only a very minor rate of growth of the errors, a very good adjustment can be accomplished, even with a method which has a very small adjustment rate. This situation is illustrated by the experiments of Charney et al. and Jastrow and Halem (1970). Although they started with a wind field that approximated that of a randomly chosen map, they obtained a very good adjustment of the wind to the temperature field, but in order to do so a simulated time of the order of 15 days or more was needed. With observations which are alien to the numerical model, and with large mathematical and physical error growth rates, such small rates of adjustment may not be able to reduce the error to a satisfactory level. For example, Nitta and Hovermale (1967) report discouraging results from their experiments with real data. Thus, we may need a method which can be adjusted to the higher rate of error growth we can expect when using real data.

Several aspects of the Charney-Halem-Jastrow initialization procedure can be studied with this end in mind. The amount of selective damping of the highfrequency waves, which they obtained with the Matsuno time-differencing scheme, was an arbitrary choice. When seeking the optimum amount of such a selective damping, it is convenient to use a scheme which has a damping that can easily be changed as a function of a parameter of the scheme. One such variable-damping scheme, which is a generalization of the Heun and Matsuno schemes, is described in the Appendix of this paper. The optimum amount and frequency of restoration of the observed variables may depend on other details of the procedure. A number of experiments, devoted to these and other aspects of the procedure for forced initialization, are described in the following sections. These experiments also show the relative merit of the different adjustment mechanisms, and illustrate some physical properties of the simulated atmosphere.

The initialization experiments that will be described are all performed using the simulated data obtained in a numerical general circulation experiment, calculated with the UCLA two-level atmospheric general circulation model (Gates et al., 1971). In the general circulation simulation the model uses the Matsuno timedifferencing scheme for the pressure gradient terms in the equations of motion and for the mass divergence terms in the continuity equation, and the Heun timedifferencing scheme for the remaining terms of the prognostic equations. This same numerical model, usually with some modifications, is used to perform the 


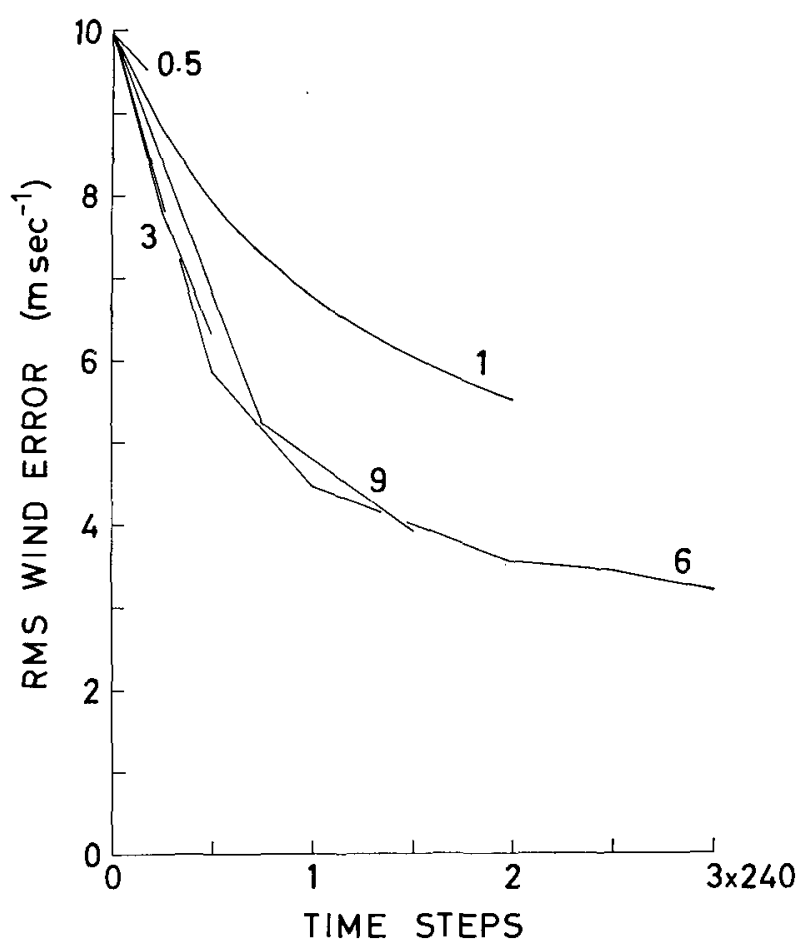

Fig. 1. Average values of the rms wind error for five single timelevel data experiments which differ only in the time amplitude of the iteration. The number next to each curve gives the iteration amplitude in hours.

initialization of the wind field. In all but one of the experiments it was assumed that the complete height field is given, with no observational errors. In the remaining case, the temperature field alone was assumed to be given.

\section{Single time-level height data, with iterative adjustment}

Initialization with data at a single time-level is simple as far as handling of the data is concerned, and yet capable of optimizing a number of features of the initialization procedure. It also helps us to interpret the experiments which use time derivatives of the height field. A disadvantage of the iterative use of the single time-level data, in numerical integration of the equations of motion, is that it requires friction to be treated abnormally, as friction is not time-reversible. The procedures and results of a number of such single timelevel data initialization experiments are described below, with the experiments grouped so as to show the dependence of the rate of error reduction and its asymptotic level on the particular features of the method.

\section{a. Time amplitude of the iteration}

Five single time-level data experiments were performed which differed only in the value of the time amplitude of the iteration. The experiments were all started from the height field of one of the general circulation experiments for which the sun had its declination and distance equal to that of 15 January; this height field will be referred to as the "control" height field. To start the experiments the wind field of this time was, however, replaced by that of 15 days earlier. The model was then run one iteration amplitude forward in time, two amplitudes backward, two amplitudes forward, and so on, for five different values of iteration amplitude. The model used for these initialization experiments differed in two ways from the model used for the general circulation experiment itself. First, the Matsuno scheme was here used for the time differencing in all of the terms of the prognostic equations and, second, the frictional force was set equal to zero. After every $3,2,3,2, \ldots$ time steps (of $6 \mathrm{~min}$ ), the current height field was replaced by a weighted average formed by taking $\frac{1}{3}$ of the current field and $\frac{2}{3}$ of the control height field. (With a 1-hr iteration amplitude, this frequency and amount of the height field restoration was found to be the best in a set of experiments with different frequencies and amounts of height field restoration.)

At the beginning of each experiment, and at every forward or backward passing through the initial time, the rms difference between the calculated and the control wind field was computed, for each of the two levels of the model. The average values of these two rms wind "errors" are shown in Fig. 1 as functions of the total number of time steps, for each of the five experiments. Here 240 time steps correspond to 1 day of simulated time. The number next to each curve gives the iteration amplitude, in hours.

Comparing the curves in Fig. 1, we see a large improvement in the rate of error reduction with increase of the iteration amplitude up to about 3-6 hr. It seems that an iteration amplitude which is of the order of one-fourth of the average time period of the inertial oscillation gives the best results. For this reason, in the remaining single time-level data experiments a time amplitude of $6 \mathrm{hr}$ is used.

The asymptotic limit of the error was not seen to noticeably depend on the time amplitude of the iteration. The experiments with 1 - and 6 -hr amplitudes were run for 16 and 7 days, respectively, and reached about the same limit of the error, $2.9 \mathrm{~m} \mathrm{sec}^{-1}$. Therefore, calculation of the wind from the height field, using a single time-step iteration amplitude, as was done by Nitta and Hovermale $(1967,1969)$ and Nitta (1969), appears to be needlessly demanding of computer time.

Finally, we can note in the 6 -hr amplitude curve that the rms wind error, when passing backward through the control time, tends to be greater than when passing forward. 


\section{b. The damping of the time-differencing scheme}

The intensity of the damping of high-frequency waves by the Matsuno time-differencing scheme is not necessarily the optimum intensity of damping. Therefore, we have investigated the dependence of the rate of wind error reduction and the asymptotic limit of the wind error on the intensity of damping of the timedifferencing scheme.

To illustrate the total effect of damping, an experiment was first carried out with the time-differencing scheme used in the general circulation model for simulation experiments; this scheme was designed to have about the minimum rate of damping and yet remain computationally stable. As described in the preceding section, it is a combination of the Heun and Matsuno schemes. Following this, some experiments were made with the scheme given in the Appendix of this report. This is a generalization of the Heun and Matsuno scheme, obtained when the time derivative in the second evaluation is computed so as to correspond to the time value of $t_{0}+a \Delta t$, where $t_{0}$ is the time at the beginning of the time step $\Delta t$, and $a$ is a variable parameter. Because a change in the parameter $a$ affects the time step allowed for linear stability, it is not practical to prescribe an arbitrary value of $a$. Instead, it is more convenient to prescribe $\Delta t$ and then compute $a$, using Eq. (13) of the Appendix.

Fig. 2 shows the results of the experiment with the combined Heun-Matsuno scheme, as well as the results of three experiments in which the time-differencing scheme given in the Appendix is applied, in the same way, to the pressure gradient, Coriolis and advection terms in the prognostic equations. In the first of the three experiments $a=1$, which makes the scheme identical to the Matsuno scheme. The time step was not changed, so that 60 time steps were contained in the 6-hr amplitude. In the second experiment, described by the full curve, the time step was chosen so as to have 65 time steps per $6 \mathrm{hr}$, which for low frequencies gives about twice the damping of the Matsuno scheme. In the third experiment, described by the dot-dashed curve, the time step was chosen to have 80 time steps per $6 \mathrm{hr}$, which for low frequencies gives about three times the damping of the Matsuno scheme. In all other aspects, all three of these experiments, as well as the one with the combined Heun-Matsuno scheme, were identical to 6-hr amplitude experiment shown in Fig. 1. In Fig. 2 and later on, however, the rms errors are plotted only when passing forward through the control time.

We see, first of all, that the combined Heun-Matsuno scheme, which has the smallest damping, was the poorest for the purpose of computing the wind by iterative forced adjustment to the height field. The schemes with progressively greater damping produced better winds. An asymptotic limit apparently exists to the improvement that can be obtained by increasing the damping, since an experiment performed with still

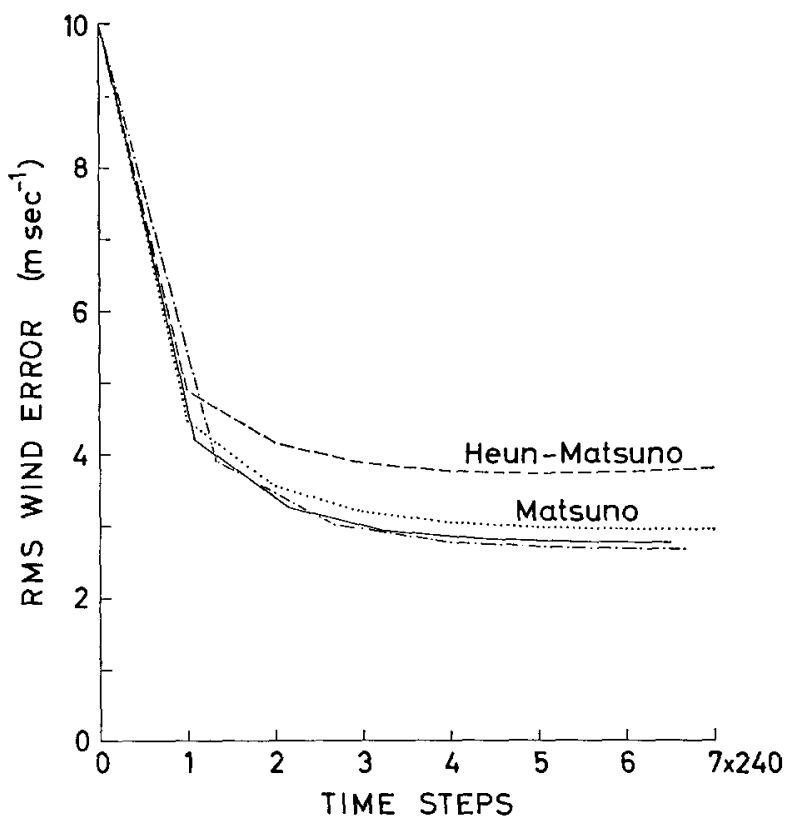

FIG. 2. Average values of the rms wind error for four single timelevel data experiments which differ only in the time-differencing scheme. The dashed curve shows the results of an experiment using a combination of the Heun and Matsuno schemes. The three remaining curves show results of experiments using that described in the Appendix. There were 60 time steps per $6 \mathrm{hr}$ in the experiment shown by the dotted curve (where the scheme was identical to that of Matsuno), 65 time steps per $6 \mathrm{hr}$ for the experiment shown by the full curve, and 80 time steps per $6 \mathrm{hr}$ for the experiment shown by the dot-dashed curve. In these three experiments the intensity of damping increases with the shortening of the time step.

stronger damping produced no further improvement in the asymptotic limit of the error.

\section{c. The effect of friction}

Several experiments were made to find whether and how friction (as used in the Mintz-Arakawa model) might reduce the asymptotic limit of the error. In this respect friction was found beneficial only when applied during the forward part of the last iteration. The only noteworthy improvement due to friction was obtained with the combined Heun-Matsuno scheme, but this improvement still left the final result poorer than those of the three experiments with more intensive timedifferencing damping. The improvement obtained in the three experiments with more intensive damping was very small, and decreased with an increase in the intensity of damping. In the experiment with 80 time steps per $6 \mathrm{hr}$, this improvement amounted only to about several percent of the asymptotic error with no friction.

\section{d. The effect of initial wind errors}

To investigate the effect of differences in the initial wind errors, three experiments were made which in 


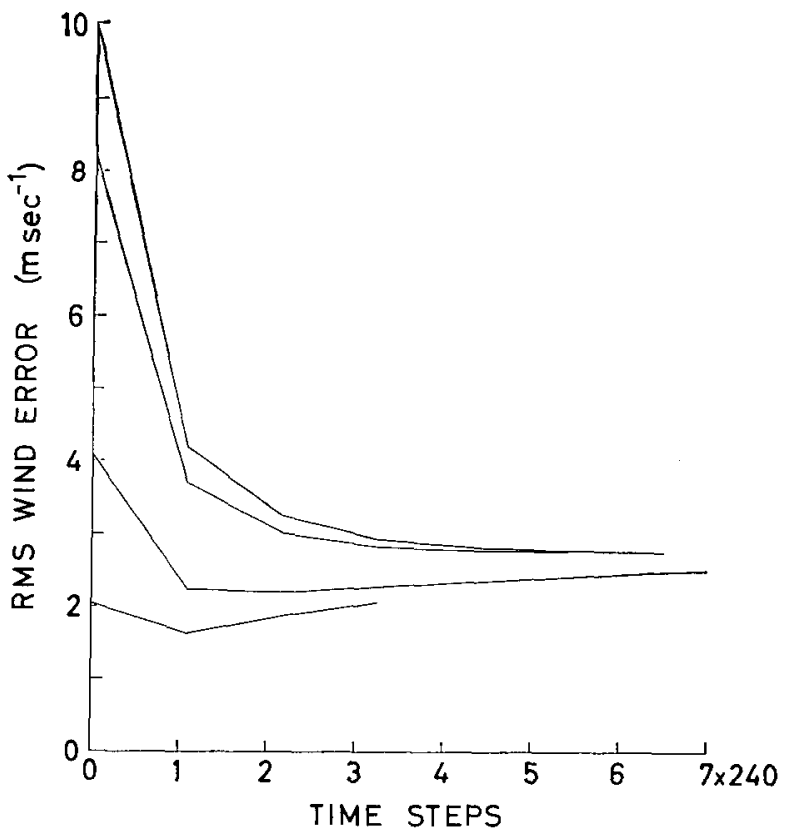

Fig. 3. Average values of the rms wind error for four single timelevel data experiments which differ only in the initial wind fields.

every respect were the same as the one shown by the full curve in Fig. 2, except for having different initial wind fields. These three experiments were started with the control wind components, to which evenly distributed random errors were superimposed, of amplitudes equal to 10,5 and $2.5 \mathrm{~m} \mathrm{sec}^{-1}$, respectively.

Fig. 3 shows a duplication of the full curve from Fig. 2 together with the results of the three experiments which were started with random wind errors. We see that starting the computation with a wind field which has sufficiently small random errors will result in more accurate winds than when starting with a randomly chosen wind field. However, for the best results, those computations should be terminated at some appropriate time when the wind field is best adjusted to the control height field. After that time, the information supplied by the better initial wind field becomes lost to a larger and larger extent.

The result of this group of experiments illustrates the danger in starting forced initialization experiments with rather small errors in the unknown fields, as, for example, was done by Nitta and Hovermale (1969) in the experiment shown in their Fig. 7. In that case, if the computation is not run long enough one can obtain an optimistic but false impression about the asymptotic limit of the error.

\section{e. Frequency and amount of the height field restoration}

A fairly large number of experiments were performed to find the optimum frequency and amount of the restoration of the height field. The main result of these experiments is illustrated by the four curves shown in
Fig. 4. In this figure the dashed and the dot-dashed curves are duplications of the dashed (combined HeunMatsuno scheme) and the dot-dashed (intensive damping with 80 time steps per $6 \mathrm{hr}$ ) curves from Fig. 2. The two full curves in Fig. 4 are obtained in the same way (the upper full curve by the combined Heun-Matsuno scheme, the lower full curve by intensive damping with 80 time steps per $6 \mathrm{hr}$ ), except that complete restoration of the height field was performed after every time step.

The four curves show that the optimum frequency and amount of the height field restoration depend on the intensity of damping of the time-differencing scheme. With small damping of the high-frequency waves, as in the combined Heun-Matsuno scheme, partial restoration every few time steps gave a better rate of adjustment than complete restoration every time step. Experiments with the combined HeunMatsuno scheme and still less frequent restoration, not shown in the figure, gave a poorer adjustment rate than the one shown by the dashed curve, which is in disagreement with an earlier finding of Charney et al. With intensive damping, as in the experiments shown by the two lower curves, complete restoration every time step was of some small advantage. The addition of friction during the forward part of the last iteration (not shown in the figure) then gave an asymptotic limit of the wind error of $2.53 \mathrm{~m} \mathrm{sec}^{-1}$ for the global average.

The experiments shown in Fig. 4 have implications for the performance of the adjustment mechanisms at work in a baroclinic numerical model. As noticed by Winninghoff (1968), the partial restoration of the height field in a barotropic model makes the adjustment rate much larger than the one obtained with a complete height restoration. In an experiment with a Lagrangian barotropic model developed by Mesinger (1971), the total time needed to reach the asymptotic limit of the error through partial restoration was reduced to about one-third of the time needed with complete restoration of the height field. This increase in the adjustment rate is due to the fact that in a barotropic atmosphere, where only external gravity waves are possible, the combined inertia-gravity wave is always faster than the pure inertia wave; thus, the adjustment proceeds faster when gravity waves are allowed to some extent. In a baroclinic atmosphere partial restoration of the height field allows both external and internal gravity waves. The slower internal waves then harm the adjustment rate to the extent which just about counteracts the benefit obtained from the faster external waves. In this way, in a baroclinic model, the adjustment rates obtained with complete and with partial height field restoration are not much different from each other.

\section{Multiple time-level height data, with iterative adjustment}

As far as observations are concerned, the adjustment to data of a single time level, described in the preceding 
section, is only a three-dimensional procedure. Forced adjustment to single time-level height data is in a way analogous to setting the time derivatives of the height field variables equal to zero, where the resulting winds should be about comparable to those obtained by solving the balance equation. Winninghoff (1968) finds them somewhat better. When using data of more than one time level, and performing a forced adjustment through numerical integration of the prognostic equations, the data assimilation is entirely four-dimensional. In such assimilation, additional information about the time derivatives of the height field is brought into the initialization procedure. This, through the pressure tendency equation and possibly also its time derivatives, puts additional constraints on the wind field and should, in principle, lead to a more accurate determination of the winds.

In a four-dimensional assimilation of data taken in a relatively short interval of time, as was the case in the experiments which will be discussed in this section, one must still expect an iterative procedure to be advisable. It allows ample time for the inertial oscillations to proceed through a number of periods. As indicated by the experiments shown in Fig. 1, this helps the rate of adjustment.

The output of the general circulation simulation

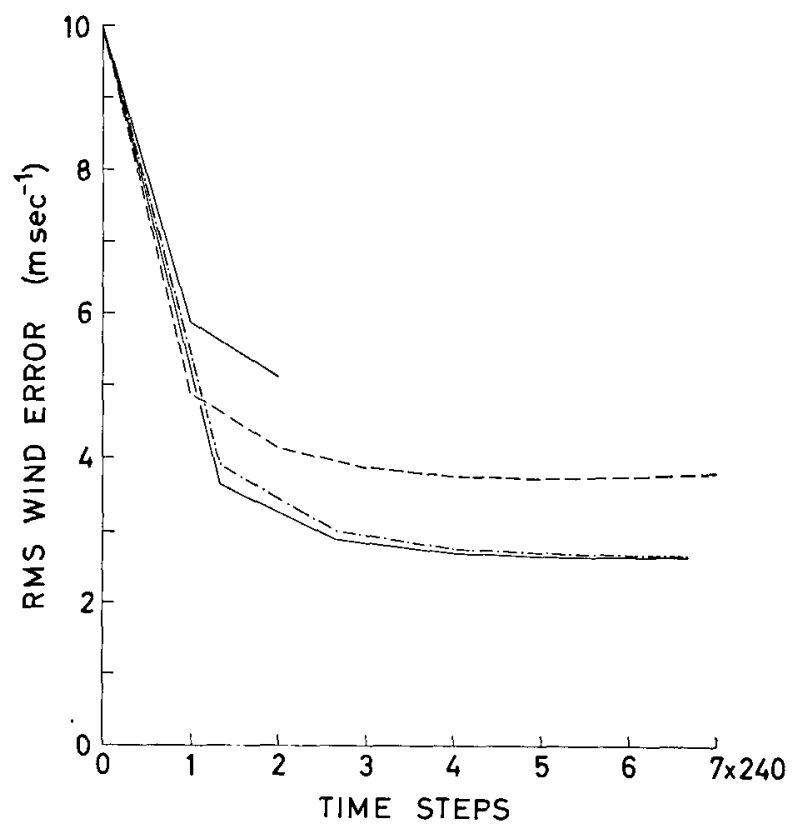

Fig. 4. Average values of the rms wind error for four single timelevel data experiments which differ in the frequency and amount of the height field restoration, and in the time differencing technique employed. The experiments shown by the dashed and the dot-dashed curve were carried out with $\frac{2}{3}$ height field restoration every $3,2,3,2, \ldots$ time steps, while the complete restoration every time step was performed in the experiments shown by the full curves. The experiments shown by the two upper curves used the combined Heun-Matsuno scheme, while the experiments shown by the two lower curves used that given in the Appendix, with 80 time steps per $6 \mathrm{hr}$.

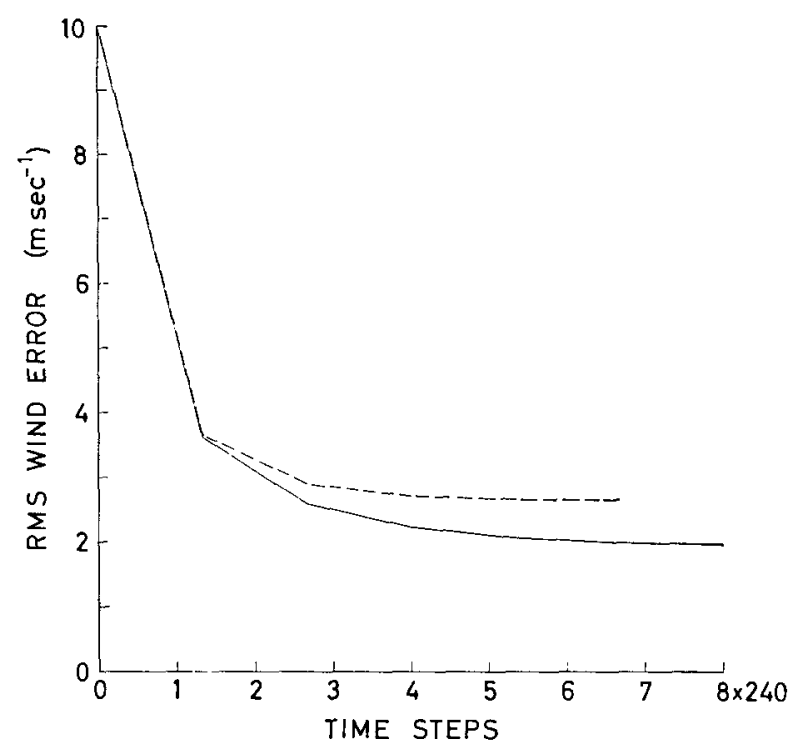

Fig. 5. Average values of the rms wind error for two experiments which differ only in the number of time levels of the height data. The dashed curve shows the results obtained in a single time-level data experiment and the full curve the results when two consecutive time levels of the data are used.

experiment with the Mintz-Arakawa model, used here for the simulated observations, was recorded on magnetic tapes at intervals of every $6 \mathrm{hr}$. The experiments which will be discussed in this section were carried out using two and three of these 6-hr simulated fields.

\section{a. Adjustment to data at two time levels}

An experiment was performed which in every respect was the same as the best of the single time-level data experiments, described by the lower of the two full curves in Fig. 4, except that data at two time levels were used. The additional height field used was the one 6-hr before the control time. The prognostic model was run $3 \mathrm{hr}$ forward in time, $12 \mathrm{hr}$ backward, $12 \mathrm{hr}$ forward, and so on, with a complete restoration of the height field being made after each time step, using linearly interpolated or extrapolated height field data. In this way, additional information on the first time derivatives of the temperature and surface pressure is brought into the initialization procedure. This, as a side advantage, relieves the computation of the adjustment to data not at its proper moment of simulated time.

The rms wind error of this two time-level data experiment, as a function of the total number of time steps, is shown by the full curve in Fig. 5. The dashed curve is a duplication of the curve describing the best of the single time-level data experiments.

Comparison of the two curves in Fig. 5 shows that a noticeable improvement in the asymptotic limit of the wind error is obtained due to information on the first time derivatives of the height field data. This improvement illustrates the superiority of the "quasi-isallobaric" 


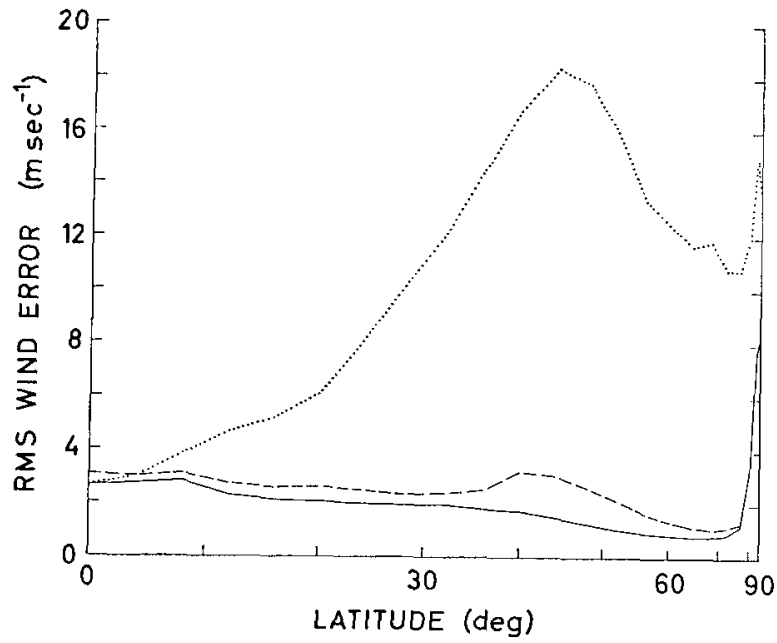

Fig. 6. Average values of the rms wind error as a function of latitude. The dotted curve shows the initial wind error, the dashed curve the error of the best adjustment when using a single time level of the data, and the full curve the error of the best adjustment when using two consecutive time levels of the data.

over the "quasi-geostrophic" wind adjustment, and appears to demonstrate the advantage of a continuous approach to the four-dimensional data assimilation problem. With friction during the forward part of the last iteration (not shown in the figure) the two timelevel data experiment gave an asymptotic limit of the error of $1.91 \mathrm{~m} \mathrm{sec}^{-1}$ for the global average.

\section{b. Adjustment to data at three time levels}

An experiment was also performed with three time levels of data: the control time, $6 \mathrm{hr}$ earlier, and $12 \mathrm{hr}$ earlier. The prognostic model was run $12 \mathrm{hr}$ backward in time, $12 \mathrm{hr}$ forward, $12 \mathrm{hr}$ backward, etc. A complete restoration of the height field was made after each time step, using linearly interpolated height field data. In this way additional information on the time change in the first time derivatives of the temperature and surface pressure is brought into the initialization procedure. The improvement in the asymptotic limit of the wind error, obtained in this three time-level data experiment, was very small: after $8 \times 240$ time steps, and friction during the forward part of the last iteration, an asymptotic limit of $1.86 \mathrm{~m} \mathrm{sec}^{-1}$ was obtained. This is only $0.05 \mathrm{~m} \mathrm{sec}^{-1}$ smaller than the error in the two time-level data experiment. On the other hand, the two time-level data experiment showed an improvement, compared to the best single time-level data experiment, of $0.62 \mathrm{~m}$ $\mathrm{sec}^{-1}$ for the global average.

Another three time-level data experiment was performed in which a parabolic time interpolation of the height field data was used, but the wind error with the parabolic interpolation of the height data was not significantly different from that of the linear interpolation experiment.

\section{Adjustment as a function of latitude}

In view of the latitude dependence of the wind-toheight and height-to-wind adjustment processes, it is of interest to consider the asymptotic limit of the wind error as a function of latitude. This is done in Fig. 6, for best single-time wind adjustments obtained with single and with two time levels of the height data.

In the figure, the abscissa scale is proportional to the sine of the latitude, and the ordinate shows the rms wind differences averaged for the two levels and the two hemispheres. The dotted curve shows the initial rms wind difference, obtained by replacing the control wind field with the one of 15 days earlier. The dashed curve shows the rms wind error of the best adjustment obtained with a single time level of the height data, and the full curve the rms wind error of the best adjustment obtained with two time levels of the height data. The curves are based on average rms values computed at intervals of $4^{\circ}$ of latitude.

The dotted curve illustrates the time variability of the wind in the simulated atmosphere: maximum variability occurs between $40^{\circ}$ and $50^{\circ}$ latitude. Except for the maximum in the polar regions, the maximum wind error for the single time-level data adjustment also occurs in this middle-latitude region. Inclusion of the data on the first time derivative of the height field into the initialization procedure reduces the wind error in all latitudes. Most significantly, it eliminates the error maximum at middle latitudes in the regions of large time variability of the height data. In these regions, the first time derivative of the height field reduces the wind error to about half of that obtained when using no time derivative. Except for the middlealtitude maximum of the single time-level data error, and the maxima of both error curves at the poles, the asymptotic limit of the wind error in both cases shows a general trend of the error decreasing with latitude.

At the equator no significant improvement of the wind error, with respect to the "random field" error of the dotted curve, occurs in either of the two experiments shown in Fig. 6. If one starts with random initial wind errors that are larger at the equator than those given by the randomly chosen wind field, then there is a reduction of the wind error at the equator due to the initialization procedure. This was observed in the experiments described in Fig. 3. For example, the experiment with an initial amplitude of the error of $10 \mathrm{~m} \mathrm{sec}^{-1}$ had an average rms initial error at the equator equal to $8.22 \mathrm{~m} \mathrm{sec}^{-1}$; at the end of the experiment this error was reduced to about half that value.

Both error curves show a large increase of the average rms wind error at the poles. The time step in the simulation experiment with the Mintz-Arakawa model was chosen so as to just about satisfy the linear stability requirement [Eq. (5) of the Appendix] in the polar regions of smallest distance between neighboring grid points; and in the present time-differencing procedure 
no shortening of the time step was attempted in order to change this situation. Thus, in polar regions, the highest frequencies are not damped by the time-differencing schemes used here. Most likely, this is the reason for the observed error maximum.

At this point it is worth noticing that the global rms area averages of the wind error, shown in all figures except Fig. 6, are heavily weighted by the relatively large errors in low-latitude regions. In middle- and high-latitude regions, as seen in Fig. 6, the method is significantly better than what is shown by the global average values of the error.

\section{Multiple time-level height data, with time- advancing adjustment}

With an increase in the time interval of the available data, there is time for an increasing number of inertial oscillations of the wind field; thus, there should occur a moment at which it becomes unnecessary to perform the adjustment through an iterative procedure, which has the disadvantage of an abnormal treatment of friction. Therefore, for sufficiently long intervals of available data, forward only ("time-advancing") running of the prognostic model should become capable of producing better adjusted wind fields than an iterative running. For this reason, experiments with timeadvancing running of the prognostic model are of interest as a further test of the procedure analyzed in the preceding sections.

Two experiments were carried out with timeadvancing adjustment, using height data at five consecutive time levels. These five levels cover the period of $24 \mathrm{hr}$ preceding the control time of the iterative experiments. The two time-advancing experiments were different from each other only in the timedifferencing scheme used. One experiment was performed using the Matsuno scheme for the pressure gradient, Coriolis and advection terms of the equations, and with time steps of $6 \mathrm{~min}$; this allows for 240 time steps in $24 \mathrm{hr}$. In the other experiment the scheme described in the Appendix was used, where there were $8 \times 240$ time steps in $24 \mathrm{hr}$; this makes the total damping of the high frequencies about equal to that imposed in the iterative experiments with multiple time-level data, described in Section 4. The two time-advancing experiments were started with the same wind fields as the multiple time-level iterative experiments. Friction was now treated in the same way as in the numerical simulation experiment. Complete restoration of the height field was again made after each time step, using linearly interpolated height field data.

At the beginning of the two experiments and at every subsequent passing through a time level of the simulated height data, the average rms difference between the calculated wind field and that of the simulation experiment was computed. These rms wind errors are shown in Fig. 7 as functions of the total number of time steps,

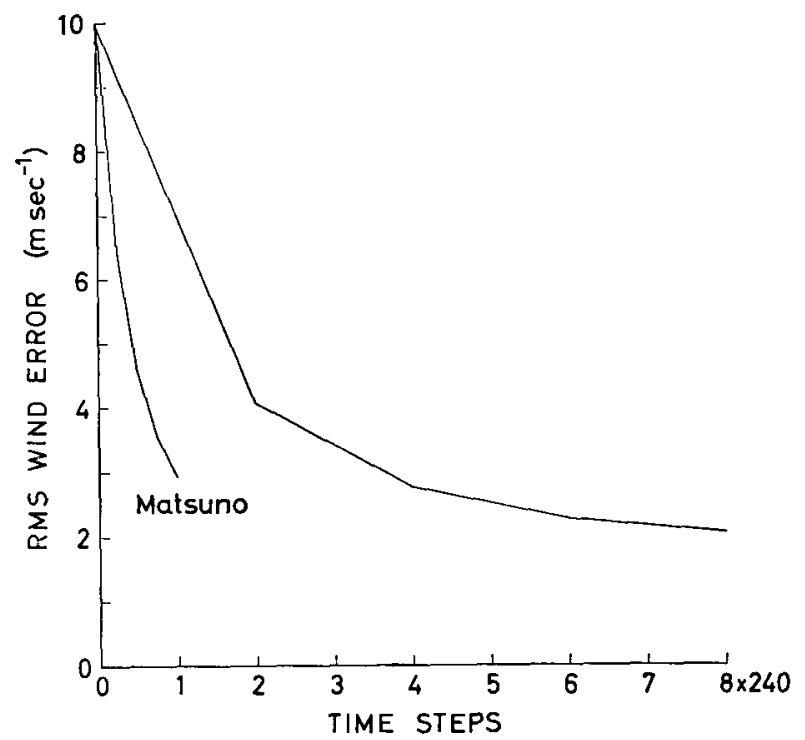

FIg. 7. Average values of the rms wind error for two experiments using five time levels $(24 \mathrm{hr}$ ) of data and time-advancing adjustment; the experiments differ only in the time-differencing technique, one uses the Matsuno scheme with time steps of 6 min and the other the scheme described in the Appendix, with time steps of $\frac{3}{4}$ of a minute.

for each of the two experiments. The steeper curve, labeled "Matsuno," shows the results of the experiment using the Matsuno scheme; the nonlabeled curve shows the results obtained in the experiment with eight times shorter time steps and more intensive damping of the high-frequency motions.

The experiment with intensive damping is seen to produce a wind field with an rms error about $30 \%$ smaller than that of the Matsuno experiment. This error, however, is still slightly greater than that of the best iterative experiment with three time levels of height data. This shows the need for a somewhat greater number of inertial oscillations of the wind field, at least when starting with a large error in the initial wind, as in present experiments.

\section{Multiple time-level temperature data, with time- advancing adjustment}

In this case the only change from the high-damping five time-level experiment of the previous section was that adjustment was made to temperature data alone. To start this experiment the wind and the surface pressure of the initial fields were replaced by those of 15 days before the control time. After each time step a complete restoration was made of just the temperature field, using linearly interpolated temperature field data.

The average rms differences between the wind fields calculated in this temperature-data experiment and the wind fields of the simulation experiment are shown in Fig. 8 by the dashed curve. This curve is also based on values computed at intervals of $6 \mathrm{hr}$. The full curve in 


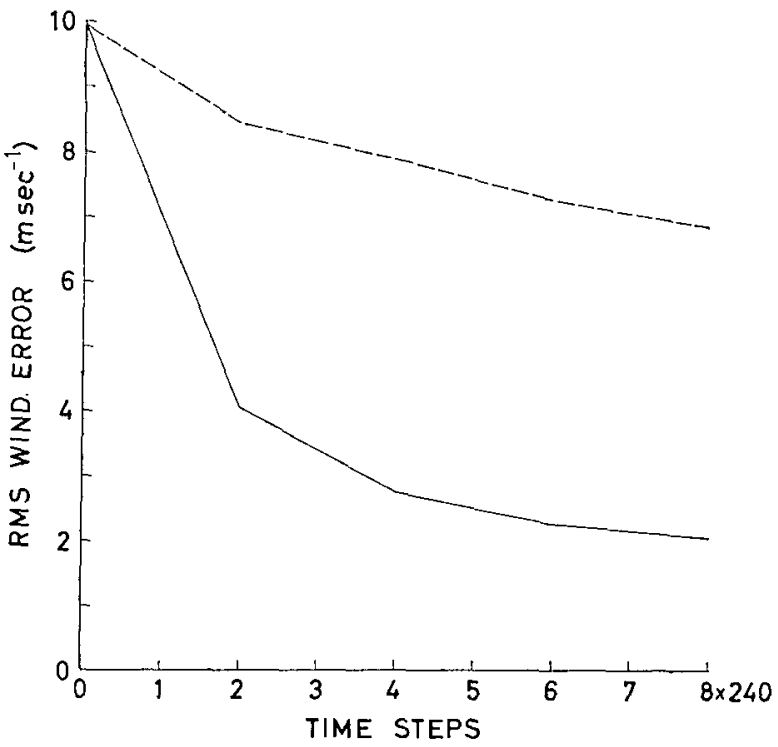

FIG. 8. Average values of the rms wind error for two experiments using five time levels $(24 \mathrm{hr})$ of data and time-advancing adjustment; the experiments differ only in the type of the data. The full curve shows the results using the complete height data (temperature as well as surface pressure); the dashed curve shows the results of an experiment using temperature data alone.

the figure is a duplication of the nonlabeled curve from Fig. 7, showing the results of the adjustment to the complete height field data.

When limited to $24 \mathrm{hr}$, the wind adjustment to temperature data alone is seen to be distinctly inferior to the adjustment to the complete height field data.

The adjustment of the surface pressure to the temperature field was also rather slow. The average rms surface pressure error, starting from the value of $7.00 \mathrm{mb}$, decreased only to $5.66 \mathrm{mb}$ in $24 \mathrm{hr}$.

\section{Summary and conclusions}

A number of aspects of the forced dynamical adjustment of the wind to the height field were experimentally studied. This was done using as observations data generated in a simulation experiment with the UCLA two-level atmospheric general circulation model. The same numerical model was used to perform the wind adjustment experiments.

A group of experiments with iterative adjustment to single time-level height data demonstrates the advantage of allowing ample time for development of inertial oscillations of the wind field. The optimum rate of adjustment appears to occur when the time amplitude of the iteration is of the order of one-fourth of the average time period of the inertial oscillations.

Considerable effort was directed to finding an optimum amount of damping in the time-differencing scheme. A scheme with variable damping was used, such that the intensity of damping of the high-frequency waves is a function of a parameter which can be changed as desired. This scheme is obtained as a generalization of those developed by Heun and Matsuno. Its properties, and the associated time-differencing procedure, are described in the Appendix. It is shown that the procedure enables an arbitrary total amount of damping to be imposed within a given interval of simulated time. Damping that is more intensive than that obtained by using the Matsuno scheme was found helpful in reducing the wind error. In single time-level data experiments, it resulted not only in a lower asymptotic limit of the wind error, but also in a greater rate of error reduction, measured per time step. This happened even though the increase in damping, for reasons of linear stability, required shorter time steps, and was thus associated with a slower progress (per time step) of the inertial oscillations.

The optimum amount and frequency of restoration of the "observed" variables was found to depend on the damping rate of the time-differencing scheme. With intensive damping, complete restoration every time step gave the most rapid rate of error reduction and the smallest asymptotic limit of the wind error. To take advantage of that property, a time interpolation of observed data was made in the experiments using more than one time level of the data.

The results obtained by forced adjustment to the height data of two and three consecutive time levels were compared with those obtained with the single time-level data. The first time derivatives of the height field variables (temperature and surface pressure) gave a substantial reduction of the wind error. In the regions of most intensive time variations of the height field, i.e., in middle latitudes, these first time derivatives resulted in a reduction of the wind error to about half of that obtained when using no time derivatives. Thus, in middle latitudes, the "quasi-isallobaric" wind adjustment is shown to be distinctly superior to the "quasigeostrophic" wind adjustment. The reduction of the wind error by use of the second time derivatives of the height field variables was, however, quite small.

Because it allows a normal treatment of friction, forward-only running of the prognostic model for sufficiently long intervals of available data should be capable of producing better adjusted wind fields than iterative procedures. The experiment with five time levels $(24 \mathrm{hr})$ of the height data illustrates the error reduction that can be obtained in such a forward running with the use of a time-differencing scheme which has an increased intensity of damping of highfrequency waves. This damping increase reduced the wind error by about $30 \%$ of that obtained with the Matsuno time-differencing scheme.

A number of possibilities exist for useful extensions of this work. These might include tests of the present procedure with simulated data of a time period $>24 \mathrm{hr}$, tests of the effect of the final asymptotic wind errors on prediction, tests with mixed height and wind data, and 
tests with real data. In addition, it appears worthwhile attempting use of the present method for regional numerical prediction, so that the variables on a fine grid are forced to adjust to those predicted on a coarse grid by a global-scale numerical forecasting model.

Acknowledgments. I wish to thank Profs. Yale Mintz and Akio Arakawa for the use of their two-level atmospheric general circulation model. They developed this model, at the University of California, Los Angeles, with the support of the Atmospheric Sciences Section, National Science Foundation, under Grant GA-1470.

Special thanks are due to Prof. Mintz, who suggested some of the experiments and helped edit the text.

\section{APPENDIX}

\section{Initialization and Filtering Characteristics of the General Two-Time-Level, Two-Derivative- Evaluation, Time-Differencing Scheme}

It appears at present that the time-differencing schemes which are able to damp high-frequency waves, such as that proposed by Matsuno (1966a), are very useful for initialization purposes. With the expected improvement in the accuracy and quantity of timecontinuous observations, of the type planned for the First GARP Global Experiment, these schemes may become essential for a satisfactory adjustment of the imbalances introduced by the assimilation of the data (see, for example, the preceding paper and the references). However, the optimum total amount of damping to be imposed in such an initialization, within a given interval of physical time, may depend on the degree to which the time changes of the observed data and of the variables in the numerical forecasting model are alien to each other. In this situation, schemes with variable damping, such that the intensity of damping is a continuous function of some parameter and can easily be changed as desired, appear most convenient.

One method which is able to damp high-frequency waves, and in which the intensity of the damping is a continuous function of some parameter, is the two-timelevel scheme with three evaluations of the time derivative which was investigated by Matsuno (1966b). Another with these properties is the three-time-level scheme with one evaluation of the time derivative (Mesinger, 1971).

Here the damping properties of the two-time-level scheme with two evaluations of the time derivative will be analyzed. In this technique the Heun and the Matsuno (two evaluation) schemes are contained as special cases. Thus, the system will be convenient for models which use the Heun or the Matsuno schemes, or combinations of the two, for time differencing during the forecast.

Let $\chi$, which can be complex, denote a dependent variable of the equations of motion, and $t$ time. Dis- regarding the dependence on other variables, the time change in $\chi$ can be described by

$$
\frac{d \chi}{d t}=f(\chi, t)
$$

Using an integer subscript to denote the number of time steps elapsed, we can write the general two-time-level, two-derivative-evaluation, finite-difference version of (1) as

$$
\left.\begin{array}{c}
\chi^{*}=\chi_{\tau}+a \Delta t f\left(\chi_{\tau}, \tau \Delta t\right) \\
\chi_{\tau+1}=\chi_{\tau}+\Delta t f\left[\chi^{*},(\tau+a) \Delta t\right]
\end{array}\right\} .
$$

When $a=0$, (2) degenerates into the forward (Euler) scheme; when $a=\frac{1}{2}$ it describes the Heun scheme, and when $a=1$ it describes the Matsuno (Euler backward) scheme.

To analyze the stability and damping properties of (2), we consider the case

$$
f(\chi, t)=i \omega \chi
$$

and define

$$
\chi_{\tau+1}=\lambda \chi_{\tau}
$$

Combining (2), (3) and (4), we obtain

$$
\lambda=1-a p^{2}+i p
$$

where

$$
p=\omega \Delta t
$$

For stability, the amplification factor

$$
|\lambda|=\left[1+(1-2 a) p^{2}+a^{2} p^{4}\right]^{\frac{1}{2}}
$$

must not be greater than 1. Thus, for the oscillation equation, (2) is stable when

$$
|p| \leqslant(2 a-1)^{\frac{1}{2}} / a .
$$

Here, we allow for the possibility of having $\Delta l<0$, that is, running the numerical model backward in time. From (5) it follows that we can have a stable range of $p$ only when $a>\frac{1}{2}$. The maximum value of the right side, and thus the maximum extent of the stability range, occurs when $a=1$ (the Matsuno scheme). With further increase in $a$, the extent of the stable (or damping) range monotonically decreases, going to zero as $a$ goes to infinity.

If one wishes to choose $\Delta t$ so as to remain inside the range in which damping increases monotonically with frequency, then one must have

$$
|p| \leqslant(2 a-1)^{\frac{1}{2}} /\left(2^{\frac{1}{a}} a\right)
$$

for all frequencies present. The right side still has the maximum value for $a=1$.

For an initialization computation, the damping properties that result from (5), and especially (6), are 
of exceptional advantage. The best alternative is to choose $\Delta t$ so as to obtain maximum damping at the expected maximum frequency, $\omega_{\max }$; in this way we use the complete high-frequency damping range in which damping monotonically increases with frequency. This is obtained when for $\omega=\omega_{\max }$ we have the equal sign in (6), that is when

$$
|\Delta t|=(2 a-1)^{\frac{1}{2}} /\left(2^{\frac{1}{a}} a \omega_{\max }\right) .
$$

We denote the value of $\Delta t$ obtained in this way for the Matsuno scheme as $\Delta t_{M}$. Then

$$
\left|\Delta t_{M}\right|=1 /\left(2^{1} \omega_{\max }\right)
$$

However, this choice of $a=1$, giving the Matsuno scheme, is arbitrary. We do not know, a priori, whether the damping obtained by the Matsuno scheme is the optimum one, and an analysis of other possibilities appears appropriate. The damping properties of (2) continuously change with the parameter $a$. One can conveniently describe such dependence with a "damping factor"

$$
\delta=1-|\lambda|
$$

which is the fractional decrease in the amplitude, per time step. When $\Delta t$ and $a$ are related as in (7), which requires that $a>\frac{1}{2}$, we obtain

$$
\delta(a, F)=1-\left[1-2\left(1-\frac{1}{2 a}\right)^{2} F^{2}\left(1-\frac{1}{2} F^{2}\right)\right]^{\frac{1}{2}},
$$

where

$$
F=\omega / \omega_{\max }
$$

is frequency as a fraction of the expected maximum frequency, $\omega_{\max }$. Thus, we expect to always have $0 \leqslant F \leqslant 1$.

From (10) we see that $\partial \delta / \partial a>0$ for any $0<F<2^{\frac{1}{2}}$ and $a>\frac{1}{2}$. Thus, by increasing the value of $a$, we increase the damping, per time step, for all the expected frequencies. In addition, $\partial(\partial \delta / \partial a) / \partial F>0$ for all $0<F<1$. Therefore, the increase in the damping increases with frequency. In the limit when $a \rightarrow \infty$, we have

$$
\delta(a, F) \rightarrow 1-\left|1-F^{2}\right|,
$$

or, for $F \leqslant 1$

$$
\delta(a, F) \rightarrow F^{2}
$$

We shall compare this limiting damping with that obtained by the Matsuno scheme. Denoting, analogously to $(8), \delta(1, F)=\delta_{M}$, or

$$
\delta_{M}=1-\left[1-\frac{1}{2} F^{2}\left(1-\frac{1}{2} F^{2}\right)\right]^{\frac{1}{2}},
$$

we have, for small $F$

$$
\delta / \delta_{M} \rightarrow 4\left(1+\frac{3}{8} F^{2}+\cdots\right),
$$

and, for $F=1$

$$
\delta / \delta_{M} \rightarrow 2\left(2+3^{\frac{1}{2}}\right) \approx 7.5 .
$$

Thus, with a large increase in the parameter $a$, as compared to the value $a=1$, we increase the damping, per time step, at the smallest frequencies, by a factor of about 4 , and damping at the highest frequencies by about 7.5. As for increasing the total amount of damping within a given interval of simulated time, one can, obviously, obtain an arbitrary total amount of damping by an appropriate shortening of the time step.

Experiments with numerical models are a straightforward way of looking for the optimum amount of damping per unit of simulated time. Some experimental results of this kind are described here. Using simulated observations, it was found that a damping more intensive than that of the Matsuno scheme can be beneficial both in increasing the speed of the forced adjustment of the wind, and in reducing the asymptotic limit of the wind error. In such experiments, in order to have the desired interval of simulated time contain an integer number of time steps, one has to define $\Delta l$, and then compute $a$ as a function of $\Delta t$. The required relation, obtained from (7) and (8), is

$$
a=\frac{1}{r^{2}}\left[1+\left(1-r^{2}\right)^{\frac{1}{2}}\right]
$$

where

$$
r=\Delta t / \Delta t_{M} \leqslant 1
$$

is the time step as a function of the one needed for the Matsuno scheme. The same formula is obtained when (5), and not (6), is used to define $|\Delta t|$ and $\left|\Delta t_{M}\right|$. In (13) only the plus sign is written in front of the square root in order to retain here only the high-damping $a>1$ possibility.

\section{REFERENCES}

Charney, J. G., M. Halem and R. Jastrow, 1969: Use of incomplete historical data to infer the present state of the atmosphere. J. Atmos. Sci., 26, 1160-1163.

Gates, W. L., E. S. Batten, A. B. Kahle and A. B. Nelson, 1971: A documentation of the Mintz-Arakawa 2-level atmospheric general circulation model. Tech. Rept., Rand Corp., Santa Monica, Calif.

Jastrow, R., and M. Halem, 1970: Simulation studies related to GARP. Bull. Amer. Meteor. Soc., 51, 490-513.

Matsuno, T., 1966a: Numerical integrations of the primitive equations by a simulated backward difference method. J. Meteor. Soc. Japan, Ser. 2, 44, 76-84.

$\longrightarrow, 1966 \mathrm{~b}$ : A finite difference scheme for time integrations of oscillatory equations with second order accuracy and sharp cut-off for high frequencies. J. Meteor. Soc. Japan, Ser. 2, $44,85-88$.

Mesinger, F., 1971 : Numerical integration of the primitive equations with a floating set of computation points: Experiments with a barotropic global model. Mon. Wea. Rev., 99, 15-29.

Miyakoda, K., and R. W. Moyer, 1968: A method of initialization for dynamical weather forecasting. Tellus, 20, 115-128.

Nitta, T., 1969: Initialization and analysis for the primitive equation model. Proc. WMO/IUGG Symp. Numerical Wea- 
ther Prediction, Tokyo, 1968, Japan Meteor. Agency, Tech. Rept. No. 67, VI-11 to VI-20.

- , and J. B. Hovermale, 1967: On analysis and initialization for the primitive forecast equations. Tech. Memo. WBTM NMC-42, Weather Bureau, Suitland, Md., $52 \mathrm{pp}$.

- , and - , 1969: A technique of objective analysis and initialization for the primitive forecast equations. Mon. Wea. $R e v .$, 97, 652-658.
Winninghoff, F. J., 1968: On the adjustment toward a geostrophic balance in a simple primitive equation model with application to the problems of initialization and objective analysis. Ph.D. thesis, Dept. Meteor., University of California, Los Angeles, $161 \mathrm{pp}$.

WMO-ICSU Joint Organizing Committee, 1969: The planning of the First GARP Global Experiment. GARP Publ. Ser. No. 3, World Meteor. Organization, Geneva, 36 pp. 